MPI-PhT/2003-21

TUM-HEP-507/03

RM3-TH/03-05

\title{
On the two-loop Yukawa corrections to the MSSM Higgs boson masses at large $\tan \beta$
}

\author{
A. Dedes ${ }^{a, 1}$, G. Degrassi ${ }^{b, 2}$, P. Slanvich ${ }^{c, d, 3}$ \\ ${ }^{a}$ Physik Department, Technische Universität München, \\ D-85748 Garching, Germany \\ ${ }^{b}$ Dipartimento di Fisica, Università di Roma Tre and \\ INFN, Sezione di Roma III, Via della Vasca Navale 84, I-00146 Rome, Italy \\ ${ }^{c}$ Institut für Theoretische Physik, Universität Karlsruhe, \\ Kaiserstrasse 12, Physikhochhaus, D-76128 Karlsruhe, Germany \\ d Max Planck Institut für Physik, \\ Föhringer Ring 6, D-80805 München, Germany
}

\begin{abstract}
We complete the effective potential calculation of the two-loop, top/bottom Yukawa corrections to the Higgs boson masses in the Minimal Supersymmetric Standard Model, by computing the $\mathcal{O}\left(\alpha_{t}^{2}+\alpha_{t} \alpha_{b}+\alpha_{b}^{2}\right)$ contributions for arbitrary values of the bottom Yukawa coupling. We also compute the corrections to the minimization conditions of the effective potential at the same perturbative order. Our results extend the existing $\mathcal{O}\left(\alpha_{t}^{2}\right)$ calculation, and are relevant in regions of the parameter space corresponding to $\tan \beta \gg 1$. We extend to the Yukawa corrections a convenient renormalization scheme, previously proposed for the $\mathcal{O}\left(\alpha_{b} \alpha_{s}\right)$ corrections, that avoids unphysically large threshold effects associated with the bottom mass and absorbs the bulk of the corrections into the one-loop expression. For large values of $\tan \beta$, the new contributions can account for a variation of several $\mathrm{GeV}$ in the lightest Higgs boson mass.
\end{abstract}

\footnotetext{
${ }^{1}$ dedes@ph.tum.de

${ }^{2}$ degrassi@fis. uniroma3.it

${ }^{3}$ slavich@mppmu.mpg.de
} 


\section{Introduction}

One of main features of the Minimal Supersymmetric Standard Model (MSSM) [1] is the prediction of the existence of at least one light Higgs boson [2]. After the conclusion of the LEP and Tevatron Run I experimental programs that reported no significant evidence for a Higgs boson, the experimental search for this particle has now become one of the major tasks of the Tevatron Run II and of the future LHC. Within the MSSM, the tree-level masses of the neutral Higgs bosons can be parameterized in terms of three input parameters: the mass of the $\mathrm{CP}$-odd Higgs $m_{A}$, the $\mathrm{Z}$ boson mass $m_{Z}$ and the ratio of the two Higgs vacuum expectation values, $\tan \beta \equiv v_{2} / v_{1}$. At tree level, at least one of the MSSM Higgs bosons is bound to be lighter than the $\mathrm{Z}$ boson, thus the failure of detecting it at LEP indicates that the MSSM could be a realistic theory only after the radiative corrections to the Higgs boson masses have been taken into account.

The radiative corrections arise from loop diagrams involving Standard Model particles and their superpartners. Although the earliest computations [3] of radiative corrections to the MSSM Higgs masses date back to the eighties, it was first realized in Ref. 4] that the inclusion of the oneloop top/stop $\mathcal{O}\left(\alpha_{t}\right)$ corrections, where $\alpha_{t}=h_{t}^{2} /(4 \pi), h_{t}$ being the superpotential top coupling, may push the light Higgs mass well above the tree-level bound. In the subsequent years, an impressive theoretical effort has been devoted to the precise determination of the MSSM Higgs masses: full one-loop computations have been provided [5, 6, leading logarithmic effects at two loops have been included via appropriate renormalization group equations [7, 8], and genuine two-loop corrections of $\mathcal{O}\left(\alpha_{t} \alpha_{s}\right)$ [9, 10, 11, 12, 13, $\mathcal{O}\left(\alpha_{t}^{2}\right)$ [9, 12, 14, and $\mathcal{O}\left(\alpha_{b} \alpha_{s}\right)$ [15] have been evaluated in the limit of zero external momentum. The tadpole corrections needed to minimize the effective potential, $V_{\text {eff }}$, have also been calculated [16] at the same perturbative orders. Furthermore, the full two-loop corrections to the MSSM effective potential have been calculated in Ref. [17, together with a first study of the effect of the two-loop corrections to the Higgs masses controlled by the electroweak gauge couplings [18.

The corrections controlled by the top Yukawa coupling are in general the most relevant ones. However, in regions of the MSSM parameter space where $\tan \beta \gg 1$ the superpotential bottom coupling $h_{b}$ may be large (we recall that, at the classical level, $h_{b} / h_{t}=\left(m_{b} / m_{t}\right) \tan \beta$ ) and the one-loop bottom/sbottom corrections of $\mathcal{O}\left(\alpha_{b}\right)$, where $\alpha_{b}=h_{b}^{2} /(4 \pi)$, can be numerically relevant and compete with those of $\mathcal{O}\left(\alpha_{t}\right)$. At the two-loop level, the evaluation of the corrections controlled by the bottom Yukawa coupling requires the inclusion of one-loop, $\tan \beta$-enhanced threshold corrections to the bottom mass [19]. If the physical bottom mass is used as input parameter in the one-loop part of the computation, potentially large $\tan \beta$-enhanced corrections appear at two loops. To address this problem, a set of renormalization prescriptions for the parameters in the bottom/sbottom sector that avoid the occurrence of unphysically large threshold effects at two loops was proposed in Ref. 15] for the $\mathcal{O}\left(\alpha_{b} \alpha_{s}\right)$ part of the corrections.

The purpose of this article is to complete the calculation of the two-loop, top/bottom Yukawa

corrections to the Higgs boson masses in the effective potential approach. Such corrections were previously computed 9, 12, 14 in the limit $h_{b} \rightarrow 0$, which is accurate enough only for moderate values of $\tan \beta$. In that limit, the two-loop Yukawa corrections to the MSSM Higgs masses are of $\mathcal{O}\left(\alpha_{t}^{2} m_{t}^{2}\right)$, which we denote as $\mathcal{O}\left(\alpha_{t}^{2}\right)$ for brevity. On the other hand, when the bottom Yukawa coupling is left arbitrary, the resulting two-loop corrections are proportional to various 
combinations of couplings and masses: e.g., we find terms of $\mathcal{O}\left(\alpha_{b}^{2} m_{t}^{2}\right)$, which might as well be interpreted as $\tan \beta$-enhanced terms of $\mathcal{O}\left(\alpha_{t} \alpha_{b} m_{b}^{2}\right)$. To simplify our notation, we will refer to all such "mixed" terms as to $\mathcal{O}\left(\alpha_{t} \alpha_{b}\right)$ corrections, and to the terms that depend only on the bottom Yukawa coupling as to $\mathcal{O}\left(\alpha_{b}^{2}\right)$ corrections. Our computation will thus provide us with the $\mathcal{O}\left(\alpha_{t}^{2}+\alpha_{t} \alpha_{b}+\alpha_{b}^{2}\right)$ corrections to the MSSM Higgs masses, extending the $\mathcal{O}\left(\alpha_{t}^{2}\right)$ results obtained in Ref. 14]. As a byproduct, we also calculate the $\mathcal{O}\left(\alpha_{t}^{2}+\alpha_{t} \alpha_{b}+\alpha_{b}^{2}\right)$ corrections to the minimization conditions of the effective potential. We express our results in the $\overline{\mathrm{DR}}$ renormalization scheme, as well as in an "on-shell" scheme which extends the prescription described in Ref. [15] to the case of the Yukawa corrections. The resulting analytical formulae are rather lengthy, thus we make them available, upon request ${ }^{1}$, in the form of a Fortran code.

The structure of this paper is the following. In section 2 we recall some general issues of the effective potential approach to the calculation of the Higgs masses. Section 3 describes our two-loop computation of the $\overline{\mathrm{DR}}$ tadpoles and $\mathrm{CP}$-odd, $\mathrm{CP}$-even Higgs mass matrices, while section 4 addresses our on-shell renormalization prescription. Numerical results are given in section 5 and in section 6 we present a short discussion of the corrections controlled by the tau Yukawa coupling as well as our conclusions.

\section{Higgs masses in the effective potential approach}

We begin our discussion by recalling some general results concerning the computation of the MSSM Higgs masses in the effective potential approach. The effective potential, which we write from the start in terms of $\overline{\mathrm{DR}}$-renormalized fields and parameters, can be decomposed as $V_{\text {eff }}=$ $V_{0}+\Delta V$, where $V_{0}$ is the tree-level scalar potential and $\Delta V$ contains the radiative corrections. Keeping only the dependence on the neutral Higgs fields $H_{1}^{0}$ and $H_{2}^{0}$, the tree-level MSSM potential reads

$$
V_{0}=\left(\mu^{2}+m_{H_{1}}^{2}\right)\left|H_{1}^{0}\right|^{2}+\left(\mu^{2}+m_{H_{2}}^{2}\right)\left|H_{2}^{0}\right|^{2}+m_{3}^{2}\left(H_{1}^{0} H_{2}^{0}+\text { h.c. }\right)+\frac{g^{2}+g^{\prime 2}}{8}\left(\left|H_{1}^{0}\right|^{2}-\left|H_{2}^{0}\right|^{2}\right)^{2},
$$

where: $\mu$ is the Higgs mass term in the superpotential (we assume it to be real, neglecting all possible CP-violating phases); $m_{H_{1}}^{2}, m_{H_{2}}^{2}$ and $m_{3}^{2}$ are soft supersymmetry-breaking masses; $g$ and $g^{\prime}$ are the $S U(2)_{L}$ and $U(1)_{Y}$ gauge couplings, respectively. The neutral Higgs fields can be decomposed into their vacuum expectation values (VEVs) plus their $\mathrm{CP}$-even and $\mathrm{CP}$-odd fluctuations as $H_{i}^{0}=\left(v_{i}+S_{i}+i P_{i}\right) / \sqrt{2}(i=1,2)$. The VEVs $v_{i}$ are determined by solving the minimization conditions of the effective potential, i.e.

$$
\left.\frac{\partial V_{\mathrm{eff}}}{\partial S_{i}}\right|_{\min }=0,\left.\quad \frac{\partial V_{\mathrm{eff}}}{\partial P_{i}}\right|_{\min }=0
$$

the second equality being automatically satisfied since we assume that $\mathrm{CP}$ is conserved. However, it is also possible to take $v_{1}$ and $v_{2}$ as input parameters, or equivalently $v^{2} \equiv v_{1}^{2}+v_{2}^{2}$ and $\tan \beta \equiv v_{2} / v_{1}$, where $v^{2}$ is related to the squared running mass of the $Z$ boson through $m_{Z}^{2}=$ $\left(g^{2}+g^{\prime 2}\right) v^{2} / 4$. In this case, the minimization conditions of $V_{\text {eff }}$ can be translated into conditions

\footnotetext{
${ }^{1}$ E-mail: slavich@mppmu.mpg.de
} 
on $\mu^{2}$ and $m_{3}^{2}$ :

$$
\begin{aligned}
\mu^{2} & =-\frac{m_{Z}^{2}}{2}+\frac{m_{H_{1}}^{2}+\Sigma_{1}-\left(m_{H_{2}}^{2}+\Sigma_{2}\right) \tan ^{2} \beta}{\tan ^{2} \beta-1}, \\
m_{3}^{2} & =\frac{m_{Z}^{2}}{2} \sin 2 \beta+\frac{1}{2} \tan 2 \beta\left(m_{H_{1}}^{2}-m_{H_{2}}^{2}+\Sigma_{1}-\Sigma_{2}\right),
\end{aligned}
$$

where the "tadpoles" $\Sigma_{1}$ and $\Sigma_{2}$ are defined as

$$
\left.\Sigma_{i} \equiv \frac{1}{v_{i}} \frac{\partial \Delta V}{\partial S_{i}}\right|_{\min }
$$

In the effective potential approach, the mass matrices for the neutral $\mathrm{CP}$-odd and $\mathrm{CP}$-even Higgs bosons can be approximated by

$$
\left(\mathcal{M}_{P}^{2}\right)_{i j}^{\mathrm{eff}}=\left.\frac{\partial^{2} V_{\mathrm{eff}}}{\partial P_{i} \partial P_{j}}\right|_{\text {min }}, \quad\left(\mathcal{M}_{S}^{2}\right)_{i j}^{\mathrm{eff}}=\left.\frac{\partial^{2} V_{\mathrm{eff}}}{\partial S_{i} \partial S_{j}}\right|_{\min } .
$$

Exploiting the minimization conditions of the effective potential, Eq. (2), the CP-odd mass matrix can be written as

$$
\left(\mathcal{M}_{P}^{2}\right)_{i j}^{\mathrm{eff}}=-m_{3}^{2} \frac{v_{1} v_{2}}{v_{i} v_{j}}-\delta_{i j} \Sigma_{i}+\left.\frac{\partial^{2} \Delta V}{\partial P_{i} \partial P_{j}}\right|_{\min } .
$$

$\left(\mathcal{M}_{P}^{2}\right)^{\text {eff }}$ has a single non-vanishing eigenvalue that, in the approximation of zero external momentum, can be identified with the squared physical mass of the $A$ boson. We denote it as $\bar{m}_{A}^{2}=\widehat{m}_{A}^{2}+\Delta m_{A}^{2}$, where $\widehat{m}_{A}^{2}=-2 m_{3}^{2} / \sin 2 \beta$ is the squared running mass of the $A$ boson. The $\mathrm{CP}$-even mass matrix can in turn be decomposed as

$$
\left(\mathcal{M}_{S}^{2}\right)^{\text {eff }}=\left(\mathcal{M}_{S}^{2}\right)^{0, \text { eff }}+\left(\Delta \mathcal{M}_{S}^{2}\right)^{\text {eff }}
$$

where the first term in the sum is the tree-level mass matrix expressed in terms of $m_{Z}$ and $\bar{m}_{A}$ :

$$
\left(\mathcal{M}_{S}^{2}\right)^{0, \mathrm{eff}}=\left(\begin{array}{cc}
m_{Z}^{2} c_{\beta}^{2}+\bar{m}_{A}^{2} s_{\beta}^{2} & -\left(m_{Z}^{2}+\bar{m}_{A}^{2}\right) s_{\beta} c_{\beta} \\
-\left(m_{Z}^{2}+\bar{m}_{A}^{2}\right) s_{\beta} c_{\beta} & m_{Z}^{2} s_{\beta}^{2}+\bar{m}_{A}^{2} c_{\beta}^{2}
\end{array}\right),
$$

( $c_{\beta} \equiv \cos \beta, s_{\beta} \equiv \sin \beta$ and so on), while the second term contains the radiative corrections:

$$
\left(\Delta \mathcal{M}_{S}^{2}\right)_{i j}^{\mathrm{eff}}=\left.\frac{\partial^{2} \Delta V}{\partial S_{i} \partial S_{j}}\right|_{\min }-\left.(-1)^{i+j} \frac{\partial^{2} \Delta V}{\partial P_{i} \partial P_{j}}\right|_{\min } .
$$

It is clear from Eqs. (17)-(10) that, in order to make contact with the physical $A$ mass, the effective potential should be computed as a function of both $\mathrm{CP}$-even and $\mathrm{CP}$-odd fields.

Since $V_{\text {eff }}$ generates one-particle-irreducible Green's functions at vanishing external momentum, it is clear that the effective potential approach neglects the momentum-dependent effects in the Higgs self-energies. The complete computation of the physical masses of the $\mathrm{CP}-$ even Higgs bosons, $m_{h}$ and $m_{H}$, and of the CP-odd Higgs boson, $m_{A}$, requires the full, momentumdependent two-point functions. A detailed discussion of the correspondence between the effective potential approach and the full computation has been presented in Ref. 14. Here we just notice 
that the main conclusions presented in that paper regarding the $\mathcal{O}\left(\alpha_{t} \alpha_{s}\right)$ and $\mathcal{O}\left(\alpha_{t}^{2}\right)$ corrections apply also to $\mathcal{O}\left(\alpha_{t} \alpha_{b}+\alpha_{b}^{2}\right)$ corrections discussed here. Namely, the two-loop $\mathcal{O}\left(\alpha_{t} \alpha_{b}+\alpha_{b}^{2}\right)$ corrections to the lightest Higgs eigenvalue are fully accounted for by the two-loop effective potential evaluation of $m_{h}$ supplemented by known momentum-dependent one-loop contributions, and the same is true for $m_{H}$ when $m_{A}$ is not too large. Instead, if $m_{A}>m_{t}$ a full two-loop $\mathcal{O}\left(\alpha_{t} \alpha_{b}+\alpha_{b}^{2}\right)$ computation of $m_{H}$ requires additional momentum-dependent two-loop contributions, neglected by the effective potential calculation, that have not been computed so far.

\section{Computation of the two-loop Yukawa corrections}

We shall now describe our two-loop computation of the tadpoles $\Sigma_{i}$, the $A$-boson mass correction $\Delta m_{A}^{2}$ and the matrix $\left(\Delta \mathcal{M}_{S}^{2}\right)^{\text {eff }}$, including terms controlled by the top and/or the bottom Yukawa couplings. The computation is consistently performed in the gaugeless limit, i.e. by setting to zero all the gauge couplings, and by keeping $h_{t}$ and $h_{b}$ as the only non-vanishing Yukawa couplings. In this limit, the tree-level (field-dependent) spectrum of the MSSM simplifies considerably: gauginos and Higgsinos do not mix; charged and neutral Higgsinos combine into Dirac spinors with degenerate mass eigenvalues $|\mu|^{2}$; the only massive SM fermions are the top and bottom quarks, while all other fermions and gauge bosons have vanishing masses; the only sfermions with non-vanishing couplings are the stop and sbottom squarks; the lightest CP-even Higgs boson, $h$, is massless, and the same is true for the Goldstone bosons; all the remaining Higgs states, $\left(H, A, H^{ \pm}\right)$, have degenerate mass eigenvalues $m_{A}^{2}$. The tree-level mixing angle in the $\mathrm{CP}$-even sector is just $\alpha=\beta-\pi / 2$.

The renormalization of the effective potential is performed according to the lines of Ref. [16], i. e. we express $V_{\text {eff }}$, from the beginning, in terms of $\overline{\mathrm{DR}}$-renormalized fields and parameters. In practice, this amounts to dropping all the divergent terms in $\Delta V$ and replacing the twoloop integrals $I\left(m_{1}^{2}, m_{2}^{2}, m_{3}^{2}\right)$ and $J\left(m_{1}^{2}, m_{2}^{2}\right)$ (see e. g. Ref. [16] for the definitions) with their "subtracted" counterparts $\hat{I}$ and $\hat{J}$, first introduced in Ref. [20. Alternatively, we could follow the procedure of Refs. 13, 14]: express $\Delta V$ in terms of bare parameters and then renormalize the derivatives of $\Delta V$ (i. e. the tadpoles and the corrections to the Higgs masses), checking explicitly the cancellation of the divergent terms. The general formulae for the tadpoles and the corrections to the Higgs masses would look slightly more complicated in the latter case. However, we have checked that the two renormalization procedures lead to the same final result, as they should.

According to Eqs. (5), (7) and (10), the tadpoles and the corrections to the Higgs mass matrices can be computed by taking the derivatives of $\Delta V$ with respect to the $\mathrm{CP}$-even and $\mathrm{CP}$-odd fields, evaluated at the minimum of $V_{\text {eff }}$. Following the strategy of Refs. [13, 14, we compute $\Delta V$ in terms of a set of field-dependent parameters (masses and angles), and use the chain rule to express the corrections in terms of derivatives of $\Delta V$ with respect to those parameters. In each sector, the field-dependent parameters can be chosen as

$$
m_{q}, m_{\tilde{q}_{1}}^{2}, m_{\tilde{q}_{2}}^{2}, \quad \bar{\theta}_{\tilde{q}}, \varphi_{q}, \widetilde{\varphi}_{q}, \quad(q=t, b),
$$

where: $m_{q}$ and $m_{\tilde{q}_{i}}^{2}$ are the quark and squark masses; $\bar{\theta}_{\tilde{q}}$ is the field-dependent squark mixing angle, defined in such a way that $0 \leq \bar{\theta}_{\tilde{q}}<\pi / 2$ (to be contrasted with the usual field-independent mixing angle $\theta_{\tilde{q}}$, such that $\left.-\pi / 2 \leq \theta_{\tilde{q}}<\pi / 2\right) ; \varphi_{q}$ is the phase in the complex quark mass; $\widetilde{\varphi}_{q}$ 
is the phase in the off-diagonal element of the squark mass matrix. For the explicit Higgs field dependence of these parameters, see Refs. [13, 14. In the expression of $\Delta V$ relevant to the $\mathcal{O}\left(\alpha_{t}^{2}\right)$ corrections (i. e., with $h_{b}$ set to zero), the top and stop phases always combine in the difference $\varphi_{t}-\widetilde{\varphi}_{t}$, so that a convenient choice for the field-dependent parameter is $c_{\varphi_{t}-\tilde{\varphi}_{t}} \equiv \cos \left(\varphi_{t}-\widetilde{\varphi}_{t}\right)$. On the other hand, when both $h_{t}$ and $h_{b}$ are nonzero, as it is the case in the present computation of the $\mathcal{O}\left(\alpha_{t}^{2}+\alpha_{t} \alpha_{b}+\alpha_{b}^{2}\right)$ corrections, the situation becomes more complicated: besides the terms involving $\varphi_{t}-\widetilde{\varphi}_{t}$ and $\varphi_{b}-\widetilde{\varphi}_{b}$, we find other terms, coming from diagrams with a charged Higgs or Goldstone boson, that involve the combinations $\varphi_{t}+\widetilde{\varphi}_{b}, \varphi_{b}+\widetilde{\varphi}_{t}, \varphi_{t}+\varphi_{b}$ and $\widetilde{\varphi}_{t}+\widetilde{\varphi}_{b}$.

Exploiting the field--dependence of the various masses and angles, we get the following general formulae for the $\mathcal{O}\left(\alpha_{t}^{2}+\alpha_{t} \alpha_{b}+\alpha_{b}^{2}\right)$ corrections in the $\overline{\mathrm{DR}}$ renormalization scheme:

$$
\begin{aligned}
\left(\Delta \mathcal{M}_{S}^{2}\right)_{11}^{\mathrm{eff}} & =2 h_{b}^{2} m_{b}^{2} F_{1}^{b}+2 h_{b}^{2} A_{b} m_{b} s_{2 \theta_{b}} F_{2}^{b}+\frac{1}{2} h_{b}^{2} A_{b}^{2} s_{2 \theta_{b}}^{2} F_{3}^{b} \\
& +\frac{1}{2} h_{t}^{2} \mu^{2} s_{2 \theta_{t}}^{2} F_{3}^{t}+2 h_{t} h_{b} m_{b} \mu s_{2 \theta_{t}} F_{4}^{t}+h_{t} h_{b} \mu A_{b} s_{2 \theta_{t}} s_{2 \theta_{b}} F_{5}, \\
\left(\Delta \mathcal{M}_{S}^{2}\right)_{12}^{\mathrm{eff}} & =h_{t}^{2} \mu m_{t} s_{2 \theta_{t}} F_{2}^{t}+\frac{1}{2} h_{t}^{2} A_{t} \mu s_{2 \theta_{t}}^{2} F_{3}^{t}+h_{t} h_{b} m_{b} A_{t} s_{2 \theta_{t}} F_{4}^{t} \\
& +h_{b}^{2} \mu m_{b} s_{2 \theta_{b}} F_{2}^{b}+\frac{1}{2} h_{b}^{2} A_{b} \mu s_{2 \theta_{b}}^{2} F_{3}^{b}+h_{t} h_{b} m_{t} A_{b} s_{2 \theta_{b}} F_{4}^{b} \\
& +\frac{1}{2} h_{t} h_{b} s_{2 \theta_{t}} s_{2 \theta_{b}}\left(A_{t} A_{b}+\mu^{2}\right) F_{5}+2 h_{t} h_{b} m_{t} m_{b} F_{6}, \\
\left(\Delta \mathcal{M}_{S}^{2}\right)_{22}^{\mathrm{eff}} & =2 h_{t}^{2} m_{t}^{2} F_{1}^{t}+2 h_{t}^{2} A_{t} m_{t} s_{2 \theta_{t}} F_{2}^{t}+\frac{1}{2} h_{t}^{2} A_{t}^{2} s_{2 \theta_{t}}^{2} F_{3}^{t} \\
& +\frac{1}{2} h_{b}^{2} \mu^{2} s_{2 \theta_{b}}^{2} F_{3}^{b}+2 h_{t} h_{b} m_{t} \mu s_{2 \theta_{b}} F_{4}^{b}+h_{t} h_{b} \mu A_{t} s_{2 \theta_{t}} s_{2 \theta_{b}} F_{5}, \\
v_{1}^{2} \Sigma_{1} & =m_{t} \mu \cot \beta s_{2 \theta_{t}} F^{t}+m_{b} A_{b} s_{2 \theta_{b}} F^{b}+2 m_{b}^{2} G^{b}, \\
v_{2}^{2} \Sigma_{2} & =m_{b} \mu \tan \beta s_{2 \theta_{b}} F^{b}+m_{t} A_{t} s_{2 \theta_{t}} F^{t}+2 m_{t}^{2} G^{t}, \\
\Delta m_{A}^{2} & =-\frac{1}{c_{\beta} s_{\beta}}\left(\frac{h_{t}^{2} \mu A_{t}}{m_{\tilde{t}_{1}}^{2}-m_{\tilde{t}_{2}}^{2}} F^{t}+\frac{h_{b}^{2} \mu A_{b}}{m_{\tilde{b}_{1}}^{2}-m_{\tilde{b}_{2}}^{2}} F^{b}+2 h_{t} h_{b} F_{A}\right) .
\end{aligned}
$$

In the equations above, $A_{t}$ and $A_{b}$ are the soft supersymmetry-breaking trilinear couplings of the Higgs fields to the stop and sbottom squarks, and $s_{2 \theta_{q}} \equiv \sin 2 \theta_{\tilde{q}}(q=t, b)$ refer to the usual field-independent squark mixing angles. The functions $F_{i}^{q}(i=1,2,3,4), F_{5}, F_{6}, F^{q}, G^{q}$ and $F_{A}$ are combinations of the derivatives of $\Delta V$ with respect to the field-dependent parameters, computed at the minimum of the effective potential; their definitions are given in the appendix. It can be noticed that, as it is predictable from the form of the MSSM Lagrangian, the above results are fully symmetric with respect to the simultaneous replacements $t \leftrightarrow b$ and $H_{1} \leftrightarrow H_{2}$, the latter resulting into $\tan \beta \leftrightarrow \cot \beta, v_{1} \leftrightarrow v_{2},\left(\Delta \mathcal{M}_{S}^{2}\right)_{11}^{\text {eff }} \leftrightarrow\left(\Delta \mathcal{M}_{S}^{2}\right)_{22}^{\text {eff }}$ and $\Sigma_{1} \leftrightarrow \Sigma_{2}$.

An explicit expression of the two-loop top and bottom Yukawa contribution to $\Delta V$ can be found in Ref. 12, while the complete two-loop effective potential for the MSSM was given in the second paper of Ref. [17. However, those expressions were computed for vanishing $\mathrm{CP}-\mathrm{odd}$ 
fields, thus omitting the dependence on the phases $\varphi_{q}$ and $\widetilde{\varphi}_{q}$. Since these phases appear in $\Delta V$ in many different combinations, it is not possible to infer the general field-dependent expression of $\Delta V$ by means of simple substitutions in Eq. (D.6) of Ref. [12, as it was the case in the computation of the $\mathcal{O}\left(\alpha_{t} \alpha_{s}+\alpha_{t}^{2}\right)$ corrections ${ }^{2}$.

We worked out the general expression of the two-loop top and bottom Yukawa contribution to $\Delta V$ in terms of all the field-dependent parameters of Eq. (11). We then computed its derivatives in order to obtain explicit formulae for the various functions appearing in Eqs. (12)-(17). The use of a recursive relation for the derivatives of $I\left(m_{1}^{2}, m_{2}^{2}, m_{3}^{2}\right)$, presented in Ref. [16], helped us to keep the number of terms involved under control. However, the resulting analytical formulae are very long and we choose not to display them in print. Instead, we make them available in the form of a Fortran code.

\section{On-shell renormalization scheme and input parameters}

The results presented in the previous section are valid when the MSSM input parameters are expressed in the $\overline{\mathrm{DR}}$ renormalization scheme. This way of presenting the results is convenient for analyzing models that predict, via the MSSM renormalization group equations, the low-energy $\overline{\mathrm{DR}}$ values of the parameters in terms of a set of boundary conditions assigned at some scale $M_{\text {GUT }}$ much larger than the weak scale (see Refs. 21, 22] for a list of public codes that are commonly used in this kind of analyses, and Ref. [23] for a comparison among them). General low-energy analyses of the MSSM, however, do not refer to boundary conditions at high scales, and are usually performed in terms of parameters with a more direct physical interpretation, such as pole masses and appropriately defined mixing angles in the squark sector. Such an approach requires modifications of our two-loop results, induced by the variation of the parameters entering the one-loop corrections when moving from the $\overline{\mathrm{DR}}$ scheme to a different scheme (for a generic parameter $x$, we define the shift from the $\overline{\mathrm{DR}}$ value $\hat{x}$ as $\delta x \equiv \hat{x}-x)$.

However, it is not always possible to find a sensible definition with a direct physical interpretation for all the relevant parameters. For example, while there is a well known physical (三 pole) definition for the masses, the so-called "On-Shell" (OS) definition, and an OS definition for the squark mixing angles can be also conceived [24], it is not clear what meaning should be assigned to an OS definition of parameters like $\left(A_{t}, A_{b}, \mu, \tan \beta\right)$. For instance, they could be related to specific physical amplitudes. However, given our present ignorance of any supersymmetric effect, such a choice does not seem particularly useful. In these cases it seems sometimes simpler to stick to a $\overline{\mathrm{DR}}$ definition.

It is rather easy to devise an OS renormalization scheme for the parameters in the top/stop sector, based on the OS prescription for the top and stop masses and the stop mixing angle and treating $A_{t}$ as a derived quantity, while retaining a $\overline{\mathrm{DR}}$ definition for $\mu$ and $\tan \beta$ (see e. g. Refs. 13, 14]). Instead, some additional care is required in the choice of an OS scheme for the parameters in the bottom/sbottom sector, due to the potentially large one-loop threshold corrections [19, proportional to $\tan \beta$, that contribute to the pole bottom mass. For example, a definition of $A_{b}$ in terms of the OS bottom and sbottom masses and sbottom mixing angle, similar to the definition of $A_{t}$, would produce a shift $\delta A_{b}$ proportional to $\tan ^{2} \beta$ [25]. When $\tan \beta$

\footnotetext{
${ }^{2}$ Also, we do not agree with Ref. [12] on the sign of the penultimate line of Eq. (D.6).
} 
is large, this would induce very large corrections to the Higgs masses at two loops, questioning the validity of the perturbative expansion.

To overcome this problem, we adopt a set of renormalization prescriptions for the parameters in the the bottom/sbottom sector, first introduced in Ref. 15] for the case of the strong corrections, that avoid the occurrence of unphysically large threshold effects and at the same time enforce other desirable properties such as the decoupling of heavy particles, the infrared finiteness and gauge-independence. Generalizing these prescriptions to the case of the Yukawa corrections, and combining them with the usual prescriptions for the top/stop parameters [14, we obtain a convenient OS renormalization scheme for the $\mathcal{O}\left(\alpha_{t} \alpha_{b}+\alpha_{b}^{2}\right)$ part of the corrections to the Higgs masses. Since the corrections controlled by the bottom Yukawa coupling can be sizeable only for large values of $\tan \beta$, we work directly in the physically relevant limit of $\tan \beta \rightarrow \infty$, i. e. $v_{1} \rightarrow 0, v_{2} \rightarrow v$.

For the OS squark masses and mixing angles, top quark mass and electroweak parameter $v \equiv\left(\sqrt{2} G_{\mu}\right)^{-1 / 2}$ we adopt the definitions

$$
\delta m_{\tilde{q}_{i}}^{2}=\Pi_{i i}^{\tilde{q}}\left(m_{\tilde{q}_{i}}^{2}\right), \quad \delta \theta_{\tilde{q}}=\frac{1}{2} \frac{\Pi_{12}^{\tilde{q}}\left(m_{\tilde{q}_{1}}^{2}\right)+\Pi_{12}^{\tilde{q}}\left(m_{\tilde{q}_{2}}^{2}\right)}{m_{\tilde{q}_{1}}^{2}-m_{\tilde{q}_{2}}^{2}}, \quad \delta m_{t}=\Sigma_{t}\left(m_{t}\right), \quad \delta v=\frac{v}{2} \frac{\Pi_{W W}^{T}(0)}{m_{W}^{2}},
$$

where $\tilde{q}=(\tilde{t}, \tilde{b})$, while $\Pi_{i j}^{\tilde{q}}\left(p^{2}\right), \Sigma_{t}(p)$ and $\Pi_{W W}^{T}\left(p^{2}\right)$ denote the real and finite parts of the self-energies of squarks, top quark and $W$ boson, respectively. Following Ref. [14, we further treat $\mu$ as a $\overline{\mathrm{DR}}$ parameter computed at a reference scale $Q_{0}=175 \mathrm{GeV}$, and $h_{t}$ and $A_{t}$ as derived quantities that can be computed by means of the tree-level formulae for $m_{t}$ and $s_{2 \theta_{t}}$, respectively. In principle, we still have to define $m_{b}, h_{b}$ and $A_{b}$. However, in the large $\tan \beta$ limit, the bottom mass is just zero, and the sbottom mixing angle becomes

$$
s_{2 \theta_{b}}=\frac{\sqrt{2} h_{b} \mu v}{m_{\tilde{b}_{1}}^{2}-m_{\tilde{b}_{2}}^{2}}
$$

which is independent of $m_{b}$ and $A_{b}$. We can thus treat $h_{b}$ as a quantity derived from the sbottom mixing, and use Eqs. (18) and (19) to obtain a prescription for $\delta h_{b}$ :

$$
\delta h_{b}=h_{b}\left(\frac{\delta m_{\tilde{b}_{1}}^{2}-\delta m_{\tilde{b}_{2}}^{2}}{m_{\tilde{b}_{1}}^{2}-m_{\tilde{b}_{2}}^{2}}+\frac{\delta s_{2 \theta_{b}}}{s_{2 \theta_{b}}}-\frac{\delta v}{v}\right) .
$$

In Ref. [15], an OS definition for the quantity $\widetilde{A}_{b} \equiv h_{b} A_{b}$, or equivalently for $\delta A_{b}=\left(\delta \widetilde{A}_{b}-\right.$ $\left.\delta h_{b} A_{b}\right) / h_{b}$, was proposed in terms of the $\left(\widetilde{b}_{1} \widetilde{b}_{2}^{*} A\right)$ proper vertex $i \Lambda_{12 A}\left(p_{1}^{2}, p_{2}^{2}, p_{A}^{2}\right)$ for the case of the strong corrections. A generalization of that definition that can also encompass the Yukawa corrections is given by

$$
\begin{aligned}
\delta \widetilde{A}_{b} & =-\frac{i}{\sqrt{2}}\left[\Lambda_{12 A}\left(m_{\tilde{b}_{1}}^{2}, m_{\tilde{b}_{1}}^{2}, 0\right)+\Lambda_{12 A}\left(m_{\tilde{b}_{2}}^{2}, m_{\tilde{b}_{2}}^{2}, 0\right)\right] \\
& +\frac{\widetilde{A}_{b}}{2}\left[\frac{\Pi_{11}^{\tilde{b}}\left(m_{\tilde{b}_{1}}^{2}\right)-\Pi_{11}^{\tilde{b}}\left(m_{\tilde{b}_{2}}^{2}\right)}{m_{\tilde{b}_{1}}^{2}-m_{\tilde{b}_{2}}^{2}}+\frac{\Pi_{22}^{\tilde{b}}\left(m_{\tilde{b}_{1}}^{2}\right)-\Pi_{22}^{\tilde{b}}\left(m_{\tilde{b}_{2}}^{2}\right)}{m_{\tilde{b}_{1}}^{2}-m_{\tilde{b}_{2}}^{2}}+\frac{\Pi_{A A}\left(m_{\tilde{b}_{1}}^{2}\right)-\Pi_{A A}\left(m_{\tilde{b}_{2}}^{2}\right)}{m_{\tilde{b}_{1}}^{2}-m_{\tilde{b}_{2}}^{2}}\right] .
\end{aligned}
$$


Having fully specified our OS renormalization prescriptions in the limit $\tan \beta \rightarrow \infty$, physically relevant for the $\mathcal{O}\left(\alpha_{t} \alpha_{b}+\alpha_{b}^{2}\right)$ corrections, we can proceed to obtain formulae for the $\mathrm{CP}$-even Higgs masses in our OS scheme and merge them with the known $\mathcal{O}\left(\alpha_{t}^{2}\right)$ OS results [14 that contain an explicit dependence on $\tan \beta$. This can be done in three steps: first, we take the limit of $\tan \beta \rightarrow \infty, m_{b} \rightarrow 0$ in the general $\overline{\mathrm{DR}}$ results for the $\mathcal{O}\left(\alpha_{t}^{2}+\alpha_{t} \alpha_{b}+\alpha_{b}^{2}\right)$ part of $\left(\Delta \mathcal{M}_{S}^{2}\right)^{\text {eff }}$; then we add the contributions due to the shifts of the parameters entering the one-loop corrections (this requires the computation of the $\mathcal{O}\left(\alpha_{t}+\alpha_{b}\right)$ part of the counterterms in the large $\tan \beta$ limit); finally, we subtract from this results the pure $\mathcal{O}\left(\alpha_{t}^{2}\right)$ part which, being relevant for all values of $\tan \beta$, must instead be computed separately with the formulae of Ref. [14]. Notice that we do not encounter any terms that blow up when we take the limit of large $\tan \beta$ in the $\overline{\mathrm{DR}}$ results: unphysically large contributions could only be introduced by hand, as the result of a poor choice of the renormalization conditions for the parameters in the bottom/sbottom sector.

We discuss now the parameters that we will actually use as inputs of our calculation. In particular, although we have used Eqs. (19)-(20) to define an OS bottom Yukawa coupling $h_{b}$ through the sbottom mixing, we still need to exploit the experimental information on the bottom mass in order to obtain the $\overline{\mathrm{DR}}$ running coupling $\hat{h}_{b}$. The OS coupling will then be computed through the relation $h_{b}=\hat{h}_{b}-\delta h_{b}$. Following Ref. [15], we define the running coupling $\hat{h}_{b}$ at the reference scale $Q_{0}=175 \mathrm{GeV}$ to be

$$
\hat{h}_{b} \equiv h_{b}\left(Q_{0}\right)_{\mathrm{MSSM}}^{\overline{\mathrm{DR}}}=\frac{\bar{m}_{b} \sqrt{2}}{v_{1}} \frac{1+\delta_{b}}{\left|1+\epsilon_{b}\right|},
$$

where: $\bar{m}_{b} \equiv m_{b}\left(Q_{0}\right)_{\mathrm{SM}}^{\overline{\mathrm{DR}}}=2.74 \pm 0.05 \mathrm{GeV}$ is the Standard Model bottom mass, evolved up to the scale $Q_{0}$ to take into account the resummation of the universal large QCD logarithms; $\epsilon_{b}$ contains the $\tan \beta$-enhanced threshold corrections from both the gluino-sbottom and the higgsino-stop loops (denoted as $\epsilon_{b}$ and $\epsilon_{b}^{\prime}$, respectively, in Eqs. (25) and (26) of Ref. [15]); $\delta_{b}$ contains the residual threshold corrections that are not enhanced by $\tan \beta$. Notice that, as shown in Ref. [26], keeping $\epsilon_{b}$ in the denominator of Eq. (22) allows to resum the tan $\beta$-enhanced threshold corrections to all orders in the perturbative expansion. On the other hand, there is no preferred way of including the threshold corrections parametrized by $\delta_{b}$, whose effect on the value of $\hat{h}_{b}$ is anyway very small. Neglecting all the terms controlled by the electroweak gauge couplings, $\epsilon_{b}$ reads

$$
\begin{aligned}
\epsilon_{b}= & -\frac{2 \alpha_{s}}{3 \pi} \frac{m_{\tilde{g}} \mu \tan \beta}{m_{\tilde{b}_{1}}^{2}-m_{\tilde{b}_{2}}^{2}}\left[\frac{m_{\tilde{b}_{1}}^{2}}{m_{\tilde{b}_{1}}^{2}-m_{\tilde{g}}^{2}} \ln \frac{m_{\tilde{b}_{1}}^{2}}{m_{\tilde{g}}^{2}}-\frac{m_{\tilde{b}_{2}}^{2}}{m_{\tilde{b}_{2}}^{2}-m_{\tilde{g}}^{2}} \ln \frac{m_{\tilde{b}_{2}}^{2}}{m_{\tilde{g}}^{2}}\right] \\
& -\frac{\alpha_{t}}{4 \pi} \frac{A_{t} \mu \tan \beta}{m_{\tilde{t}_{1}}^{2}-m_{\tilde{t}_{2}}^{2}}\left[\frac{m_{\tilde{t}_{1}}^{2}}{m_{\tilde{t}_{1}}^{2}-\mu^{2}} \ln \frac{m_{\tilde{t}_{1}}^{2}}{\mu^{2}}-\frac{m_{\tilde{t}_{2}}^{2}}{m_{\tilde{t}_{2}}^{2}-\mu^{2}} \ln \frac{m_{\tilde{t}_{2}}^{2}}{\mu^{2}}\right] .
\end{aligned}
$$

It appears from Eq. (22) that $\hat{h}_{b}$ blows up when $\epsilon_{b}$ approaches -1 , in which case the correct value of the bottom mass cannot be reproduced with $\hat{h}_{b}$ in the perturbative regime, and the corresponding set of MSSM parameters must be discarded. It can also be noticed from Eq. (23) that, since we take $m_{\tilde{g}}>0$, for $A_{t}>0\left(A_{t}<0\right)$ the $\mathcal{O}\left(\alpha_{s}\right)$ and $\mathcal{O}\left(\alpha_{t}\right)$ contributions enter $\epsilon_{b}$ with the same (the opposite) sign. Moreover, if we take only the $\mathcal{O}\left(\alpha_{s}\right)$ part of $\epsilon_{b}$ into account, $\hat{h}_{b}$ can be enhanced by the threshold correction only for large values of $m_{\tilde{g}}$ and large and positive ${ }^{3}$

\footnotetext{
${ }^{3}$ Our convention for the sign of $\mu$ is such that, e. g., the sbottom mixing parameter reads $X_{b}=A_{b}+\mu \tan \beta$.
} 
values of $\mu$, whereas, when we include the $\mathcal{O}\left(\alpha_{t}\right)$ part, $\hat{h}_{b}$ can be enhanced also for small values of $m_{\tilde{g}}$ and large and negative values of both $\mu$ and $A_{t}$.

For the top/stop sector, we take as input the current central value of the top pole mass, $m_{t}=174.3 \mathrm{GeV}$ [27], and the parameters $\left(m_{Q, \tilde{t}}, m_{U}, A_{t}\right)$ that can be derived by rotating the diagonal matrix of the OS stop masses by the angle $\theta_{\tilde{t}}$, defined in Eq. (18). Concerning the sbottom sector, additional care is required, because of our non-trivial definition of $h_{b}$ and of the fact that, at one loop, the parameter $m_{Q, \tilde{b}}$ entering the sbottom mass matrix differs from the corresponding stop parameter $m_{Q, \tilde{t}}$ by a finite shift [25]. We start by computing the renormalized coupling $h_{b}$ as given by Eq. (201) and (22). Then we compute $m_{Q, \tilde{b}}$ following the prescription of Ref. [25]. Finally, we use the parameters $h_{b}$ and $m_{Q, \tilde{b}}$ to compute the actual values of the OS sbottom masses and mixing angle. Concerning the $A$-boson mass, which enters the treelevel mass matrix for the $\mathrm{CP}$-even Higgses, we take as input the physical mass $m_{A}$, dropping the distinction between $m_{A}$ and the effective potential mass $\bar{m}_{A}$ (this amounts to neglecting the effect of the uncomputed momentum-dependent two-loop corrections). The renormalization of the $Z$-boson mass, whose numerical value we take equal to the physical mass $m_{Z}=91.187$ $\mathrm{GeV}$, does not affect the $\mathcal{O}\left(\alpha_{t} \alpha_{b}+\alpha_{b}^{2}\right)$ corrections. The remaining numerical inputs are the OS electroweak parameter $v=246.218 \mathrm{GeV}$ and the strong coupling constant, which we fix as $\alpha_{s}\left(Q_{0}\right)=0.108$.

\section{$5 \quad$ Numerical results}

We are now ready to discuss the numerical effect of our two-loop corrections. In the previous sections we have discussed how to express our results in either the $\overline{\mathrm{DR}}$ renormalization scheme or an OS scheme suitably chosen to separate the genuine two-loop corrections from the threshold corrections in the relation between $m_{b}$ and $h_{b}$. Our $\overline{\mathrm{DR}}$ results for the two-loop corrections to the Higgs masses and to the electroweak symmetry breaking conditions can be easily implemented in the existing codes [21, 22] that compute the MSSM mass spectrum from a set of unified parameters at the scale $M_{G U T}$. A study of the implications of our results in the framework of gravity (mSUGRA), gauge (GMSB) or anomaly (AMSB) mediated supersymmetry breaking models goes beyond the scope of this paper, and will appear elsewhere [28]. In the following discussion, we will adopt a low-energy point of view and assume that the various input parameters are related, when possible, to physical quantities. To this aim, we will make use of the OS renormalization scheme presented in section 4 . We recall that, although our OS prescription is defined in the limit $\tan \beta \rightarrow \infty$, the corrections have an indirect dependence on $\tan \beta$ coming from the input value for $\hat{h}_{b}$, see Eq. (22).

In Figs. 1h (upper panel) and 1b (lower panel) we show the light Higgs mass $m_{h}$ as a function of $\tan \beta$, for $m_{A}=120 \mathrm{GeV}$ and $m_{A}=250 \mathrm{GeV}$, respectively. The other input parameters are chosen as $A_{t}=1 \mathrm{TeV}, A_{b}=2 \mathrm{TeV}, \mu=m_{Q, \tilde{t}}=m_{U}=m_{D}=m_{\tilde{g}}=1 \mathrm{TeV}$. In each plot, the longdashed curve corresponds to the value of $m_{h}$ obtained at $\mathcal{O}\left(\alpha_{t}+\alpha_{t} \alpha_{s}+\alpha_{t}^{2}\right)$, i. e. by including only the one- and two-loop corrections controlled by the top Yukawa coupling; the dot-dashed curve includes in addition the one-loop $\mathcal{O}\left(\alpha_{b}\right)$ corrections, controlled by the bottom Yukawa coupling ${ }^{4}$;

\footnotetext{
${ }^{4}$ In the calculation of the one-loop $\mathcal{O}\left(\alpha_{t}\right)$ and $\mathcal{O}\left(\alpha_{b}\right)$ corrections we include the effects proportional to $m_{Z}^{2}$ and the momentum corrections as in [5].
} 

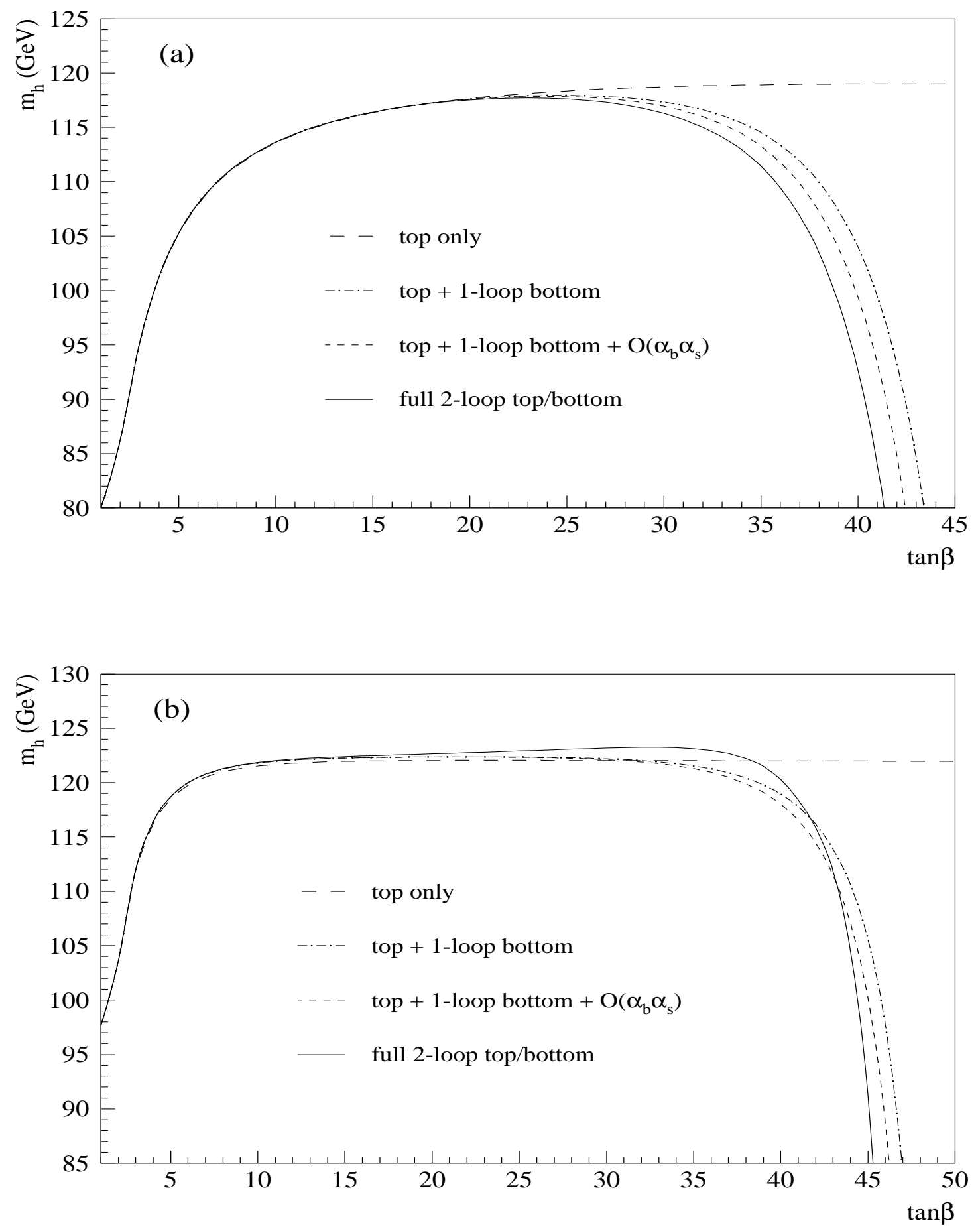

Figure 1: The mass $m_{h}$ as a function of $\tan \beta$, for $m_{A}=120 \mathrm{GeV}$ (upper panel) or $250 \mathrm{GeV}$ (lower panel). The other input parameters are $A_{t}=1 \mathrm{TeV}, A_{b}=2 \mathrm{TeV}, \mu=m_{Q, \tilde{t}}=m_{U}=$ $m_{D}=m_{\tilde{g}}=1 \mathrm{TeV}$. The meaning of the different curves is explained in the text. 


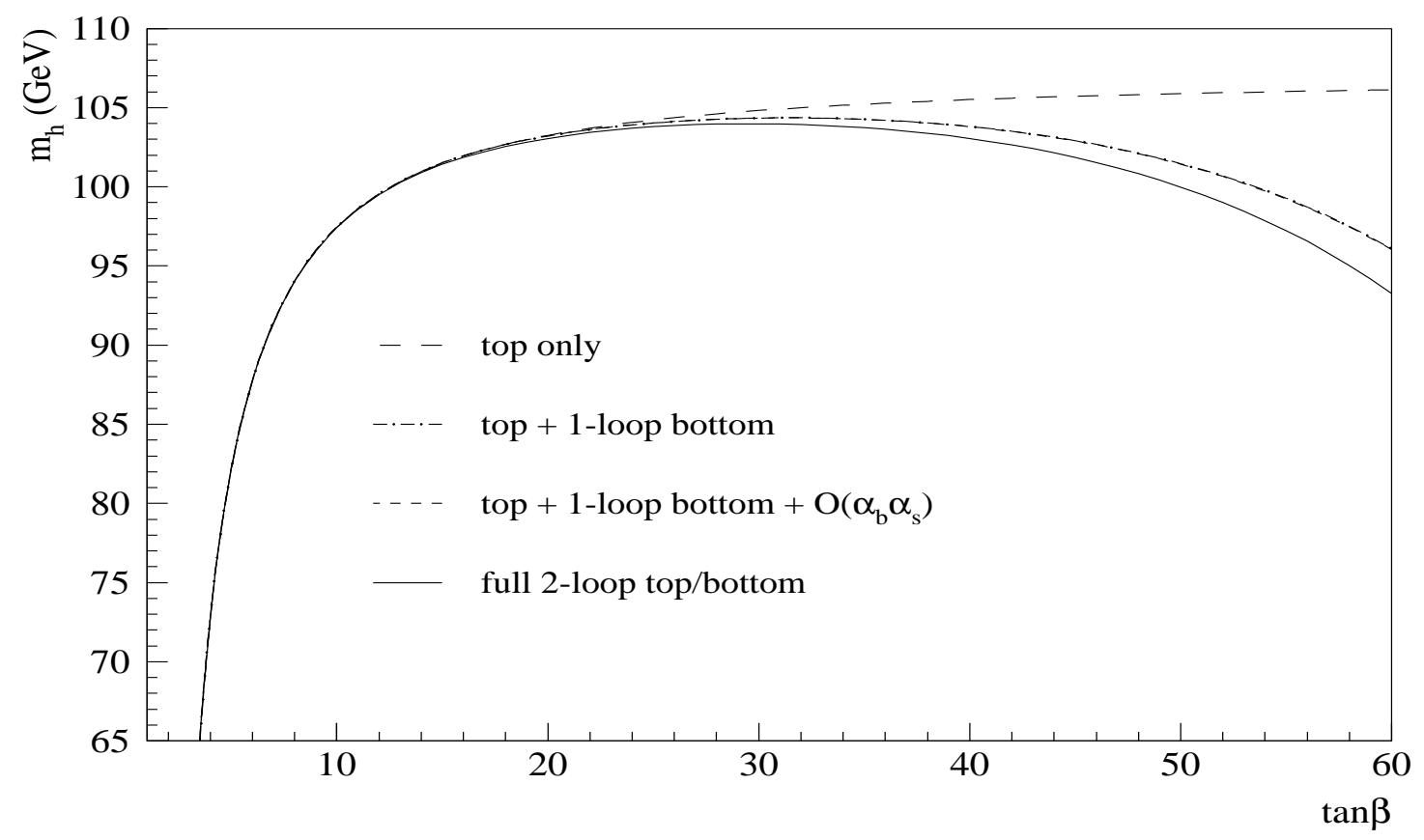

Figure 2: The mass $m_{h}$ as a function of $\tan \beta$, for $m_{A}=120 \mathrm{GeV}, \mu=A_{t}=-2 \mathrm{TeV}, A_{b}=-3$ $\mathrm{TeV}, m_{Q, \tilde{t}}=m_{U}=m_{D}=1 \mathrm{TeV}$ and $m_{\tilde{g}}=200 \mathrm{GeV}$. The meaning of the different curves is explained in the text.

the short-dashed curve includes the two-loop $\mathcal{O}\left(\alpha_{b} \alpha_{s}\right)$ corrections computed in Ref. [15]; finally, the solid curve corresponds to the full two-loop Yukawa computation of $m_{h}$, i. e. it includes also the $\mathcal{O}\left(\alpha_{t} \alpha_{b}+\alpha_{b}^{2}\right)$ corrections discussed in the previous sections. We can see from Figs. 17 and 10 that the corrections controlled by the top Yukawa coupling depend very weakly on $\tan \beta$ when the latter is large. On the other hand, the $\mathcal{O}\left(\alpha_{b}\right)$ corrections lower considerably $m_{h}$ when $\tan \beta$ increases. Concerning the two-loop corrections controlled by the bottom Yukawa coupling, the comparison between the dot-dashed and short-dashed curves shows that the $\mathcal{O}\left(\alpha_{b} \alpha_{s}\right)$ corrections amount to a small fraction of the $\mathcal{O}\left(\alpha_{b}\right)$ ones, but they can still lower $m_{h}$ by several $\mathrm{GeV}$ when $\tan \beta$ is large. The comparison between the short-dashed and solid curves shows that the effect of the $\mathcal{O}\left(\alpha_{t} \alpha_{b}+\alpha_{b}^{2}\right)$ corrections can also amount to several $\mathrm{GeV}$ when $\tan \beta$ is large. From Fig. 17 we see that, when $m_{A}$ is small and the correction to $m_{h}$ is mainly driven by $\left(\Delta \mathcal{M}_{S}^{2}\right)_{11}$, the $\mathcal{O}\left(\alpha_{t} \alpha_{b}+\alpha_{b}^{2}\right)$ corrections enter with the same sign as the $\mathcal{O}\left(\alpha_{b} \alpha_{s}\right)$ corrections, reducing further the value of $m_{h}$. On the other hand, Fig. 10 shows that for larger values of $m_{A}$, when the correction to $m_{h}$ is sensitive to $\left(\Delta \mathcal{M}_{S}^{2}\right)_{22}$, the new corrections account for an increase in $m_{h}$ of a few $\mathrm{GeV}$ at moderately large values of $\tan \beta$ (i. e., $\tan \beta \approx 30-40$ ). This is basically due to a positive contribution to $\left(\Delta \mathcal{M}_{S}^{2}\right)_{22}$ coming from the $\mathcal{O}\left(\alpha_{t} \alpha_{b}\right)$ part of corrections. When $\tan \beta$ takes on larger values, however, the overall effect of the $\mathcal{O}\left(\alpha_{t} \alpha_{b}+\alpha_{b}^{2}\right)$ corrections to $m_{h}$ turns again to negative.

It is interesting to realize that the $\mathcal{O}\left(\alpha_{t} \alpha_{b}+\alpha_{b}^{2}\right)$ corrections can be sizeable also for parameter choices that make the $\mathcal{O}\left(\alpha_{b} \alpha_{s}\right)$ corrections irrelevant. In Fig. 2 we show $m_{h}$ as a function of 


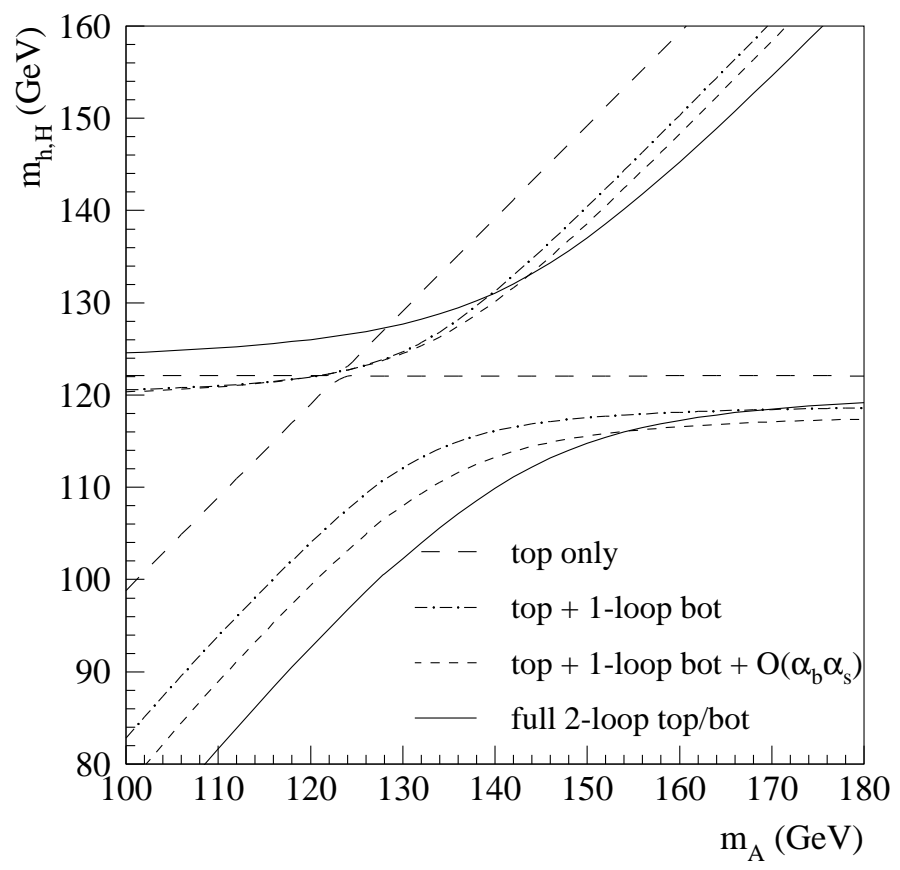

Figure 3: The masses $m_{h}$ and $m_{H}$ as a function of $m_{A}$, for $\tan \beta=40, A_{t}=1 \mathrm{TeV}, A_{b}=2 \mathrm{TeV}$, $\mu=m_{Q, \tilde{t}}=m_{U}=m_{D}=m_{\tilde{g}}=1 \mathrm{TeV}$. The meaning of the different curves is explained in the text.

$\tan \beta$, for $m_{A}=120 \mathrm{GeV}, \mu=A_{t}=-2 \mathrm{TeV}, A_{b}=-3 \mathrm{TeV}, m_{Q, \tilde{t}}=m_{U}=m_{D}=1 \mathrm{TeV}$ and $m_{\tilde{g}}=200 \mathrm{GeV}$. The meaning of the various curves is the same as in Fig. 1. Due to the small value of the gluino mass with respect to the sbottom masses, the $\mathcal{O}\left(\alpha_{b} \alpha_{s}\right)$ corrections to $m_{h}$ are negligible (in fact, the dot-dashed and short-dashed curves overlap). On the other hand, comparing the short-dashed and solid curves we see that the $\mathcal{O}\left(\alpha_{t} \alpha_{b}+\alpha_{b}^{2}\right)$ corrections can still amount to a few $\mathrm{GeV}$ when $\tan \beta$ is large enough.

Finally, Fig. 3 shows both CP-even Higgs masses, $m_{h}$ and $m_{H}$, as functions of the CP-odd Higgs mass, in the region of relatively small $m_{A}\left(100 \mathrm{GeV}<m_{A}<180 \mathrm{GeV}\right)$, for $\tan \beta=40$. The other input parameters are chosen as $A_{t}=1 \mathrm{TeV}, A_{b}=2 \mathrm{TeV} \mu=m_{Q, \tilde{t}}=m_{U}=m_{D}=m_{\tilde{g}}=1$ $\mathrm{TeV}$. The meaning of the various curves is the same as in Fig. 1 Comparing the short-dashed and solid curves we see that, for this choice of parameters, the effect of the $\mathcal{O}\left(\alpha_{t} \alpha_{b}+\alpha_{b}^{2}\right)$ corrections is particularly evident in the region where $m_{A}$ is small, and can account for variations of several $\mathrm{GeV}$ (around 5 in this example) in both $m_{h}$ and $m_{H}$.

In general, it appears from Figs. 1 3 that the two-loop $\mathcal{O}\left(\alpha_{b} \alpha_{s}\right)$ and $\mathcal{O}\left(\alpha_{t} \alpha_{b}+\alpha_{b}^{2}\right)$ corrections are usually a small fraction of the one-loop $\mathcal{O}\left(\alpha_{b}\right)$ ones. We stress that this is a desirable consequence of our renormalization prescription, which allows to set apart the $\tan \beta$-enhanced threshold corrections, resummed to all orders in the renormalized coupling $h_{b}$. If we were to adopt for the bottom/sbottom sector the same renormalization prescription that we use for the top/stop sector, the dependence on $\tan \beta$ of the one-loop corrections would be smoother, but very large corrections would appear at two loops, questioning the validity of the perturbative expansion. 
To conclude this section, we notice that our knowledge of the general formulae for the corrections to the $\mathrm{CP}$-even Higgs mass matrix in the $\overline{\mathrm{DR}}$ scheme allows us to estimate the uncertainty connected with the fact that we take the limit of $\tan \beta \rightarrow \infty, m_{b} \rightarrow 0$ in the corresponding OS results. In the numerical examples considered above we find that, in the regions where the corrections are sizeable, the $\overline{\mathrm{DR}}$ results for the $\mathcal{O}\left(\alpha_{b} \alpha_{s}\right)$ part of $\left(\Delta \mathcal{M}_{S}^{2}\right)^{\text {eff }}$ vary by less than $20 \%$ when the limit $\tan \beta \rightarrow \infty, m_{b} \rightarrow 0$ is taken. The $\overline{\mathrm{DR}}$ results for the $\mathcal{O}\left(\alpha_{t} \alpha_{b}+\alpha_{b}^{2}\right)$ part of the corrections vary instead by less than $10 \%$. We can assume that similar variations occurr also in the corresponding OS results, which leads to shifts in $m_{h}$ typically smaller than $1 \mathrm{GeV}$.

\section{Conclusions and discussion}

In this paper we computed the $\mathcal{O}\left(\alpha_{t} \alpha_{b}+\alpha_{b}^{2}\right)$ corrections to the MSSM neutral Higgs boson masses and to the minimization conditions of the MSSM effective potential. Such corrections are relevant when the ratio of the Higgs VEVs, $\tan \beta$, is large. Combined with the previously computed $\mathcal{O}\left(\alpha_{t} \alpha_{s}\right)$ [13], $\mathcal{O}\left(\alpha_{t}^{2}\right)$ [14] and $\mathcal{O}\left(\alpha_{b} \alpha_{s}\right)$ [15] corrections to the neutral Higgs masses, and with the corresponding corrections to the minimization conditions of the effective potential [16], these results provide us with a complete computation of the leading two-loop corrections controlled by the top and bottom Yukawa couplings.

Using the formalism of the effective potential, we obtained complete analytic expressions for the momentum-independent part of the $\mathcal{O}\left(\alpha_{t}^{2}+\alpha_{t} \alpha_{b}+\alpha_{b}^{2}\right)$ corrections, valid for arbitrary values of the MSSM input parameters when the latter are expressed in the $\overline{\mathrm{DR}}$ renormalization scheme. We also discussed an OS renormalization prescription for the parameters of the bottom/sbottom sector in the physically relevant limit of large $\tan \beta$. Such prescription, first introduced in Ref. [15] for the case of the $\mathcal{O}\left(\alpha_{b} \alpha_{s}\right)$ corrections, allows to separate the large threshold corrections appearing in the relation between $h_{b}$ and the pole bottom mass from the genuine two-loop effects. Finally, we discussed the numerical impact of our results in a few representative examples, showing that, for large values of $\tan \beta$, the $\mathcal{O}\left(\alpha_{t} \alpha_{b}+\alpha_{b}^{2}\right)$ corrections can induce variations in the Higgs masses of the order of a few GeV. Since our analytic expressions, both in the $\overline{\mathrm{DR}}$ and the OS schemes, are too long to be useful if explicitly written on paper, we choose to make them available in the form of a Fortran code.

Although the terms controlled by the top and bottom Yukawa couplings undoubtedly account for the bulk of the two-loop corrections, several pieces are still missing for a complete twoloop computation of the MSSM Higgs masses. When $\tan \beta$ is large, the corrections controlled by the tau Yukawa coupling $h_{\tau}$ might in principle be non-negligible. In the approximation of neglecting the electroweak gauge couplings, the only two-loop corrections involving the tau Yukawa coupling are those of $\mathcal{O}\left(\alpha_{b} \alpha_{\tau}\right)$ and those of $\mathcal{O}\left(\alpha_{\tau}^{2}\right)$, where $\alpha_{\tau}=h_{\tau}^{2} /(4 \pi)$. While the mixed $\mathcal{O}\left(\alpha_{b} \alpha_{\tau}\right)$ corrections would require a dedicated computation ${ }^{5}$, explicit formulae for the $\mathcal{O}\left(\alpha_{\tau}^{2}\right)$ corrections can be obtained from the formulae of Refs. [14, 16] for the purely $\mathcal{O}\left(\alpha_{t}^{2}\right)$ corrections, with the replacements $t \rightarrow \tau, \tilde{b}_{L} \rightarrow \tilde{\nu}_{\tau}, N_{c} \rightarrow 1$ and $H_{1} \leftrightarrow H_{2}$ [the latter resulting into $\tan \beta \leftrightarrow \cot \beta, v_{1} \leftrightarrow v_{2},\left(\Delta \mathcal{M}_{S}^{2}\right)_{11}^{\text {eff }} \leftrightarrow\left(\Delta \mathcal{M}_{S}^{2}\right)_{22}^{\text {eff }}$ and $\Sigma_{1} \leftrightarrow \Sigma_{2}$ ]. If the input parameters are given in the OS scheme, a suitable definition of $\delta A_{\tau}$ is required in order to avoid introducing $\tan \beta$-enhanced terms in the two-loop part of the result. Anyway, we find that the $\mathcal{O}\left(\alpha_{\tau}^{2}\right)$

\footnotetext{
${ }^{5}$ We thank A. Brignole for drawing our attention on this point.
} 
corrections to the Higgs masses are in general very small compared with those controlled by the bottom Yukawa coupling. Besides the hierarchy between $m_{b}$ and $m_{\tau}$, the suppression of the tau corrections is motivated by the absence of color enhancements, and by the fact that the only $\tan \beta$-enhanced threshold corrections to the relation between $h_{\tau}$ and $m_{\tau}$ are those controlled by the electroweak gauge couplings.

A full two-loop determination of the MSSM Higgs masses will require going beyond the gaugeless limit and the effective potential approximation, i. e. including both the corrections controlled by the electroweak gauge couplings and the effect of the momentum-dependent part of the Higgs self-energies. It can also be expected that, among the three-loop corrections, at least those involving the top Yukawa coupling affect the Higgs masses in a non-negligible way. In Ref. 18] the two-loop, zero-momentum electroweak corrections have been computed numerically in a representative scenario, and found to yield a shift in the lightest Higgs boson mass $m_{h}$ of about $1 \mathrm{GeV}$ with respect to the result obtained in the gaugeless approximation. In Ref. [29], the theoretical uncertainty in the prediction for $m_{h}$ arising from the combined effect of the missing two-loop corrections and the leading three-loop corrections has been estimated to be around 3 $\mathrm{GeV}$.

If the MSSM is a viable theory for physics at the weak scale, a light Higgs boson will be discovered either at the Tevatron or at the LHC. Subsequently, its properties will be determined with high precision at a future linear collider: for example, the predicted experimental accuracy in the determination of $m_{h}$ at TESLA is about $50 \mathrm{MeV}$ [30. It is thus clear that further effort will be required in the coming years, in order to improve the accuracy of the theoretical predictions up to the level required to compare with the experimental results expected at the next generation of colliders.

\section{Acknowledgments}

We would like to thank A. Brignole, A. Pilaftsis and F. Zwirner for useful comments and discussions, and T. Hahn for help in producing the Fortran routines. This work was partially supported by the European Community's Human Potential Programmes HPRN-CT-2000-00148 (Across the Energy Frontier) and HPRN-CT-2000-00149 (Collider Physics).

\section{Appendix}

We present here the expressions for the functions $F_{i}^{t}(i=1,2,3,4), F_{5}, F_{6}, F^{t}, G^{t}$ and $F_{A}$, appearing in Eqs. (12)-(17), in terms of derivatives of the $\overline{\mathrm{DR}}$-renormalized $\Delta V$, computed at the minimum of $V_{\text {eff }}$ :

$$
\begin{aligned}
F_{1}^{t} & =\frac{\partial^{2} \Delta V}{\left(\partial m_{t}^{2}\right)^{2}}+\frac{\partial^{2} \Delta V}{\left(\partial m_{\tilde{t}_{1}}^{2}\right)^{2}}+\frac{\partial^{2} \Delta V}{\left(\partial m_{\tilde{t}_{2}}^{2}\right)^{2}}+2 \frac{\partial^{2} \Delta V}{\partial m_{t}^{2} \partial m_{\tilde{t}_{1}}^{2}}+2 \frac{\partial^{2} \Delta V}{\partial m_{t}^{2} \partial m_{\tilde{t}_{2}}^{2}}+2 \frac{\partial^{2} \Delta V}{\partial m_{\tilde{t}_{1}}^{2} \partial m_{\tilde{t}_{2}}^{2}} \\
& +\frac{1}{4 m_{t}^{4}}\left(\frac{\partial \Delta V}{\partial c_{\varphi_{t}+\varphi_{b}}}+z_{t} \frac{\partial \Delta V}{\partial c_{\varphi_{t}-\tilde{\varphi}_{t}}}+z_{b} \frac{\partial \Delta V}{\partial c_{\varphi_{t}+\tilde{\varphi}_{b}}}\right)
\end{aligned}
$$




$$
\begin{aligned}
& F_{2}^{t}=\frac{\partial^{2} \Delta V}{\left(\partial m_{\tilde{t}_{1}}^{2}\right)^{2}}-\frac{\partial^{2} \Delta V}{\left(\partial m_{\tilde{t}_{2}}^{2}\right)^{2}}+\frac{\partial^{2} \Delta V}{\partial m_{t}^{2} \partial m_{\tilde{t}_{1}}^{2}}-\frac{\partial^{2} \Delta V}{\partial m_{t}^{2} \partial m_{\tilde{t}_{2}}^{2}} \\
& -\frac{4 c_{2 \theta_{t}}^{2}}{m_{\tilde{t}_{1}}^{2}-m_{\tilde{t}_{2}}^{2}}\left(\frac{\partial^{2} \Delta V}{\partial c_{2 \bar{\theta}_{t}}^{2} \partial m_{t}^{2}}+\frac{\partial^{2} \Delta V}{\partial c_{2 \bar{\theta}_{t}}^{2} \partial m_{\tilde{t}_{1}}^{2}}+\frac{\partial^{2} \Delta V}{\partial c_{2 \bar{\theta}_{t}}^{2} \partial m_{\tilde{t}_{2}}^{2}}\right)-\frac{z_{t}}{s_{2 \theta_{t}}^{2} m_{t}^{2}\left(m_{\tilde{t}_{1}}^{2}-m_{\tilde{t}_{2}}^{2}\right)} \frac{\partial \Delta V}{\partial c_{\varphi_{t}-\tilde{\varphi}_{t}}}, \\
& F_{3}^{t}=\frac{\partial^{2} \Delta V}{\left(\partial m_{\tilde{t}_{1}}^{2}\right)^{2}}+\frac{\partial^{2} \Delta V}{\left(\partial m_{\tilde{t}_{2}}^{2}\right)^{2}}-2 \frac{\partial^{2} \Delta V}{\partial m_{\tilde{t}_{1}}^{2} \partial m_{\tilde{t}_{2}}^{2}}-\frac{2}{m_{\tilde{t}_{1}}^{2}-m_{\tilde{t}_{2}}^{2}}\left(\frac{\partial \Delta V}{\partial m_{\tilde{t}_{1}}^{2}}-\frac{\partial \Delta V}{\partial m_{\tilde{t}_{2}}^{2}}\right) \\
& +\frac{16 c_{2 \theta_{t}}^{2}}{\left(m_{\tilde{t}_{1}}^{2}-m_{\tilde{t}_{2}}^{2}\right)^{2}}\left(c_{2 \theta_{t}}^{2} \frac{\partial^{2} \Delta V}{\left(\partial c_{2 \bar{\theta}_{t}}^{2}\right)^{2}}+2 \frac{\partial \Delta V}{\partial c_{2 \bar{\theta}_{t}}^{2}}\right)-\frac{8 c_{2 \theta_{t}}^{2}}{m_{\tilde{t}_{1}}^{2}-m_{\tilde{t}_{2}}^{2}}\left(\frac{\partial^{2} \Delta V}{\partial c_{2 \bar{\theta}_{t}}^{2} \partial m_{\tilde{t}_{1}}^{2}}-\frac{\partial^{2} \Delta V}{\partial c_{2 \bar{\theta}_{t}}^{2} \partial m_{\tilde{t}_{2}}^{2}}\right) \\
& +\frac{4 z_{t}}{s_{2 \theta_{t}}^{4}\left(m_{\tilde{t}_{1}}^{2}-m_{\tilde{t}_{2}}^{2}\right)^{2}}\left(\frac{\partial \Delta V}{\partial c_{\varphi_{t}-\tilde{\varphi}_{t}}}+\frac{\partial \Delta V}{\partial c_{\varphi_{b}+\tilde{\varphi}_{t}}}+z_{b} \frac{\partial \Delta V}{\partial c_{\tilde{\varphi}_{t}+\tilde{\varphi}_{b}}}\right), \\
& F_{4}^{t}=\frac{\partial^{2} \Delta V}{\partial m_{\tilde{t}_{1}}^{2} \partial m_{b}^{2}}+\frac{\partial^{2} \Delta V}{\partial m_{\tilde{t}_{1}}^{2} \partial m_{\tilde{b}_{1}}^{2}}+\frac{\partial^{2} \Delta V}{\partial m_{\tilde{t}_{1}}^{2} \partial m_{\tilde{b}_{2}}^{2}}-\frac{\partial^{2} \Delta V}{\partial m_{\tilde{t}_{2}}^{2} \partial m_{b}^{2}}-\frac{\partial^{2} \Delta V}{\partial m_{\tilde{t}_{2}}^{2} \partial m_{\tilde{b}_{1}}^{2}}-\frac{\partial^{2} \Delta V}{\partial m_{\tilde{t}_{2}}^{2} \partial m_{\tilde{b}_{2}}^{2}} \\
& -\frac{4 c_{2 \theta_{t}}^{2}}{m_{\tilde{t}_{1}}^{2}-m_{\tilde{t}_{2}}^{2}}\left(\frac{\partial^{2} \Delta V}{\partial m_{\tilde{b}_{1}}^{2} \partial c_{2 \bar{\theta}_{t}}^{2}}+\frac{\partial^{2} \Delta V}{\partial m_{\tilde{b}_{2}}^{2} \partial c_{2 \bar{\theta}_{t}}^{2}}+\frac{\partial^{2} \Delta V}{\partial m_{b}^{2} \partial c_{2 \bar{\theta}_{t}}^{2}}\right)-\frac{z_{t}}{s_{2 \theta_{t}}^{2} m_{b}^{2}\left(m_{\tilde{t}_{1}}^{2}-m_{\tilde{t}_{2}}^{2}\right)} \frac{\partial \Delta V}{\partial c_{\varphi_{b}+\tilde{\varphi}_{t}}}, \\
& F_{5}=\frac{\partial^{2} \Delta V}{\partial m_{\tilde{t}_{1}}^{2} \partial m_{\tilde{b}_{1}}^{2}}-\frac{\partial^{2} \Delta V}{\partial m_{\tilde{t}_{1}}^{2} \partial m_{\tilde{b}_{2}}^{2}}-\frac{\partial^{2} \Delta V}{\partial m_{\tilde{t}_{2}}^{2} \partial m_{\tilde{b}_{1}}^{2}}+\frac{\partial^{2} \Delta V}{\partial m_{\tilde{t}_{2}}^{2} \partial m_{\tilde{b}_{2}}^{2}} \\
& -\frac{4 c_{2 \theta_{t}}^{2}}{m_{\tilde{t}_{1}}^{2}-m_{\tilde{t}_{2}}^{2}}\left(\frac{\partial^{2} \Delta V}{\partial m_{\tilde{b}_{1}}^{2} \partial c_{2 \bar{\theta}_{t}}^{2}}-\frac{\partial^{2} \Delta V}{\partial m_{\tilde{b}_{2}}^{2} \partial c_{2 \bar{\theta}_{t}}^{2}}\right)-\frac{4 c_{2 \theta_{b}}^{2}}{m_{\tilde{b}_{1}}^{2}-m_{\tilde{b}_{2}}^{2}}\left(\frac{\partial^{2} \Delta V}{\partial m_{\tilde{t}_{1}}^{2} \partial c_{2 \bar{\theta}_{b}}^{2}}-\frac{\partial^{2} \Delta V}{\partial m_{\tilde{t}_{2}}^{2} \partial c_{2 \bar{\theta}_{b}}^{2}}\right) \\
& +\frac{16 c_{2 \theta_{t}}^{2} c_{2 \theta_{b}}^{2}}{\left(m_{\tilde{t}_{1}}^{2}-m_{\tilde{t}_{2}}^{2}\right)\left(m_{\tilde{b}_{1}}^{2}-m_{\tilde{b}_{2}}^{2}\right)} \frac{\partial^{2} \Delta V}{\partial c_{2 \bar{\theta}_{t}}^{2} \partial c_{2 \bar{\theta}_{b}}^{2}}-\frac{4 z_{t} z_{b}}{s_{2 \theta_{t}}^{2} s_{2 \theta_{b}}^{2}\left(m_{\tilde{t}_{1}}^{2}-m_{\tilde{t}_{2}}^{2}\right)\left(m_{\tilde{b}_{1}}^{2}-m_{\tilde{b}_{2}}^{2}\right)} \frac{\partial \Delta V}{\partial c_{\tilde{\varphi}_{t}+\tilde{\varphi}_{b}}}, \\
& F_{6}=\frac{\partial^{2} \Delta V}{\partial m_{t}^{2} \partial m_{b}^{2}}+\frac{\partial^{2} \Delta V}{\partial m_{\tilde{t}_{1}}^{2} \partial m_{b}^{2}}+\frac{\partial^{2} \Delta V}{\partial m_{\tilde{t}_{2}}^{2} \partial m_{b}^{2}}+\frac{\partial^{2} \Delta V}{\partial m_{\tilde{b}_{1}}^{2} \partial m_{t}^{2}}+\frac{\partial^{2} \Delta V}{\partial m_{\tilde{b}_{2}}^{2} \partial m_{t}^{2}} \\
& +\frac{\partial^{2} \Delta V}{\partial m_{\tilde{t}_{1}}^{2} \partial m_{\tilde{b}_{1}}^{2}}+\frac{\partial^{2} \Delta V}{\partial m_{\tilde{t}_{1}}^{2} \partial m_{\tilde{b}_{2}}^{2}}+\frac{\partial^{2} \Delta V}{\partial m_{\tilde{t}_{2}}^{2} \partial m_{\tilde{b}_{1}}^{2}}+\frac{\partial^{2} \Delta V}{\partial m_{\tilde{t}_{2}}^{2} \partial m_{\tilde{b}_{2}}^{2}}-\frac{1}{4 m_{t}^{2} m_{b}^{2}} \frac{\partial \Delta V}{\partial c_{\varphi_{t}+\varphi_{b}}},
\end{aligned}
$$




$$
\begin{aligned}
F^{t} & =\frac{\partial \Delta V}{\partial m_{\tilde{t}_{1}}^{2}}-\frac{\partial \Delta V}{\partial m_{\tilde{t}_{2}}^{2}}-\frac{4 c_{2 \theta_{t}}^{2}}{m_{\tilde{t}_{1}}^{2}-m_{\tilde{t}_{2}}^{2}} \frac{\partial \Delta V}{\partial c_{2 \theta_{t}}^{2}}, \\
G^{t} & =\frac{\partial \Delta V}{\partial m_{t}^{2}}+\frac{\partial \Delta V}{\partial m_{\tilde{t}_{1}}^{2}}+\frac{\partial \Delta V}{\partial m_{\tilde{t}_{2}}^{2}}, \\
F_{A} & =\frac{1}{4 m_{t} m_{b}} \frac{\partial \Delta V}{\partial c_{\varphi_{t}+\varphi_{b}}}+\frac{4\left(A_{t} A_{b}-\mu^{2}\right)^{2} m_{t} m_{b} z_{t} z_{b}}{s_{2 \theta_{t}}^{2} s_{2 \theta_{b}}^{2}\left(m_{\tilde{t}_{1}}^{2}-m_{\tilde{t}_{2}}^{2}\right)^{2}\left(m_{\tilde{b}_{1}}^{2}-m_{\tilde{b}_{2}}^{2}\right)^{2}} \frac{\partial \Delta V}{\partial c_{\tilde{\varphi}_{t}+\tilde{\varphi}_{b}}} \\
& +\frac{m_{t} z_{t}}{s_{2 \theta_{t}}^{2} m_{b}\left(m_{\tilde{t}_{1}}^{2}-m_{\tilde{t}_{2}}^{2}\right)^{2}}\left(A_{t}^{2} \frac{\partial \Delta V}{\partial c_{\varphi_{b}+\tilde{\varphi}_{t}}}+\mu^{2} \cot ^{2} \beta \frac{\partial \Delta V}{\partial c_{\varphi_{t}-\tilde{\varphi}_{t}}}\right) \\
& +\frac{m_{b} z_{b}}{s_{2 \theta_{b}}^{2} m_{t}\left(m_{\tilde{b}_{1}}^{2}-m_{\tilde{b}_{2}}^{2}\right)^{2}}\left(A_{b}^{2} \frac{\partial \Delta V}{\partial c_{\varphi_{t}+\tilde{\varphi}_{b}}}+\mu^{2} \tan ^{2} \beta \frac{\partial \Delta V}{\partial c_{\varphi_{b}-\tilde{\varphi}_{b}}}\right) .
\end{aligned}
$$

In the above formulae, $z_{q} \equiv \operatorname{sign}\left(X_{q}\right),{ }^{6}$ where $X_{q}(q=t, b)$ is the squark mixing parameter. The functions $F_{i}^{b}, F^{b}$ and $G^{b}$ can be obtained from their top counterparts through the replacement $t \leftrightarrow b$.

\section{References}

[1] H. P. Nilles, Phys. Rept. 110 (1984) 1;

H. E. Haber and G. L. Kane, Phys. Rept. 117 (1985) 75;

A. B. Lahanas and D. V. Nanopoulos, Phys. Rept. 145 (1987) 1;

for a more recent review and further references see S. P. Martin, hep-ph/9709356.

[2] J. F. Gunion, H. E. Haber, G. L. Kane and S. Dawson, The Higgs Hunter's guide, Addison Wesley, 1990 and (errata) hep-ph/9302272 for a recent continuation see M. Carena and H. E. Haber, Prog. Part. Nucl. Phys. 50 (2003) 63 hep-ph/0208209.

[3] S. P. Li and M. Sher, Phys. Lett. B140 (1984) 339;

J. F. Gunion and A. Turski, Phys. Rev. D39 (1989) 2701; Phys. Rev. D40 (1989) 2325;

M. S. Berger, Phys. Rev. D41 (1990) 225.

[4] Y. Okada, M. Yamaguchi and T. Yanagida, Prog. Theor. Phys. 85 (1991) 1; Phys. Lett. B262, (1991) 54;

J. R. Ellis, G. Ridolfi and F. Zwirner, Phys. Lett. B257 (1991) 83; Phys. Lett. B262 (1991) 477 ;

H. E. Haber and R. Hempfling, Phys. Rev. Lett. 66 (1991) 1815.

[5] A. Brignole, Phys. Lett. B281 (1992) 284.

\footnotetext{
${ }^{6}$ Factors of $z_{t}$ were omitted in Eqs. (28)-(30) and (C2) of Ref. 13. Notice also that in Eqs. (17) and (A9) we employ a different definition of $F_{A}$ compared to the one employed in Eqs. (C1)-(C2) of Ref. [13].
} 
[6] P. H. Chankowski, S. Pokorski and J. Rosiek, Phys. Lett. B274 (1992) 191;

A. Dabelstein, Z. Phys. C67 (1995) 495 hep-ph/9409375;

D. M. Pierce, J. A. Bagger, K. T. Matchev and R. J. Zhang, Nucl. Phys. B491 (1997) 3 hep-ph/9606211.

[7] M. Carena, J. R. Espinosa, M. Quiros and C. E. Wagner, Phys. Lett. B355 (1995) 209 hep-ph/9504316;

M. Carena, M. Quiros and C. E. Wagner, Nucl. Phys. B461 (1996) 407 hep-ph/9508343;

H. E. Haber, R. Hempfling and A. H. Hoang, Z. Phys. C75 (1997) 539 hep-ph/9609331;

J. R. Espinosa and I. Navarro, Nucl. Phys. B615 (2001) 82 hep-ph/0104047.

[8] A. Pilaftsis and C. E. Wagner, Nucl. Phys. B553 (1999) 3 hep-ph/9902371;

M. Carena, J. R. Ellis, A. Pilaftsis and C. E. Wagner, Nucl. Phys. B586 (2000) 92 hep-ph/0003180; Nucl. Phys. B625 (2002) 345 hep-ph/0111245.

[9] R. Hempfling and A. H. Hoang, Phys. Lett. B331 (1994) 99 hep-ph/9401219.

[10] S. Heinemeyer, W. Hollik and G. Weiglein, Phys. Rev. D58 (1998) 091701 hep-ph/9803277;

Phys. Lett. B440 (1998) 296 hep-ph/9807423; Eur. Phys. J. C9 (1999) 343 hep-ph/9812472; Phys. Lett. B455 (1999) 179 hep-ph/9903404.

[11] R. J. Zhang, Phys. Lett. B447 (1999) 89 hep-ph/9808299;

J. R. Espinosa and R. Zhang, JHEP 0003 (2000) 026 hep-ph/9912236].

[12] J. R. Espinosa and R. Zhang, Nucl. Phys. B586 (2000) 3, revised in hep-ph/0003246 v2.

[13] G. Degrassi, P. Slavich and F. Zwirner, Nucl. Phys. B611 (2001) 403 hep-ph/0105096.

[14] A. Brignole, G. Degrassi, P. Slavich and F. Zwirner, Nucl. Phys. B631 (2002) 195 hep-ph/0112177.

[15] A. Brignole, G. Degrassi, P. Slavich and F. Zwirner, Nucl. Phys. B643 (2002) 79 hep-ph/0206101.

[16] A. Dedes and P. Slavich, Nucl. Phys. B657 (2003) 333 hep-ph/0212132.

[17] S. P. Martin, Phys. Rev. D65 (2002) 116003 hep-ph/0111209; Phys. Rev. D66 (2002) 096001 hep-ph/0206136.

[18] S. P. Martin, Phys. Rev. D67 (2003) 095012 hep-ph/0211366.

[19] T. Banks, Nucl. Phys. B303 (1988) 172;

L. J. Hall, R. Rattazzi and U. Sarid, Phys. Rev. D50 (1994) 7048 hep-ph/9306309;

R. Hempfling, Phys. Rev. D49 (1994) 6168;

M. Carena, M. Olechowski, S. Pokorski and C. E. Wagner, Nucl. Phys. B426 (1994) 269 hep-ph/9402253.

[20] C. Ford, I. Jack and D.R.T. Jones, Nucl. Phys. B387 (1992) 373; Erratum ibid. B504 (1997) 551 hep-ph/0111190. 
[21] H. Baer, F. E. Paige, S. D. Protopopescu and X. Tata, hep-ph/0001086.

B. C. Allanach, Comput. Phys. Commun. 143 (2002) 305 hep-ph/0104145;

A. Djouadi, J. L. Kneur and G. Moultaka, hep-ph/0211331;

W. Porod, Comput. Phys. Commun. 153 (2003) 275 hep-ph/0301101.

[22] S. Ambrosanio, A. Dedes, S. Heinemeyer, S. Su and G. Weiglein, Nucl. Phys. B624 (2002) 3 hep-ph/0106255.

[23] B. C. Allanach, S. Kraml and W. Porod, JHEP 0303 (2003) 016 hep-ph/0302102.

[24] A. Pilaftsis, Nucl. Phys. B504 (1997) 61 hep-ph/9702393;

J. Guasch, J. Sola and W. Hollik, Phys. Lett. B 437 (1998) 88 hep-ph/9802329;

H. Eberl, S. Kraml and W. Majerotto, JHEP 9905 (1999) 016 hep-ph/9903413;

Y. Yamada, Phys. Rev. D64 (2001) 036008 hep-ph/0103046.

[25] A. Bartl, H. Eberl, K. Hidaka, T. Kon, W. Majerotto and Y. Yamada, Phys. Lett. B402 (1997) 303 hep-ph/9701398;

H. Eberl, K. Hidaka, S. Kraml, W. Majerotto and Y. Yamada, Phys. Rev. D62 (2000) 055006 hep-ph/9912463.

[26] M. Carena, D. Garcia, U. Nierste and C. E. Wagner, Nucl. Phys. B577 (2000) 88 hep-ph/9912516;

G. Degrassi, P. Gambino and G. F. Giudice, JHEP 0012 (2000) 009 hep-ph/0009337.

[27] L. Demortier, R. Hall, R. Hughes, B. Klima, R. Roser and M. Strovink [The Top Averaging Group for the CDF and D0 Collaborations], FERMILAB-TM-2084.

[28] A. Djouadi et al., in preparation.

[29] G. Degrassi, S. Heinemeyer, W. Hollik, P. Slavich and G. Weiglein, Eur. Phys. J. C28 (2003) 133 hep-ph/0212020.

[30] J. A. Aguilar-Saavedra et al. [ECFA/DESY LC Physics Working Group Collaboration], hep-ph/0106315 